\title{
Topology optimization using a mixed formulation: An alternative way to solve pressure load problems
}

\section{Sigmund, Ole; Clausen, Peter Michael}

\section{Published in:}

Computer Methods in Applied Mechanics and Engineering

Link to article, DOI:

10.1016/j.cma.2006.09.021

Publication date:

2007

Document Version

Early version, also known as pre-print

Link back to DTU Orbit

Citation (APA):

Sigmund, O., \& Clausen, P. M. (2007). Topology optimization using a mixed formulation: An alternative way to solve pressure load problems. Computer Methods in Applied Mechanics and Engineering, 196(13-16), 18741889. https://doi.org/10.1016/j.cma.2006.09.021

\section{General rights}

Copyright and moral rights for the publications made accessible in the public portal are retained by the authors and/or other copyright owners and it is a condition of accessing publications that users recognise and abide by the legal requirements associated with these rights.

- Users may download and print one copy of any publication from the public portal for the purpose of private study or research.

- You may not further distribute the material or use it for any profit-making activity or commercial gain

- You may freely distribute the URL identifying the publication in the public portal 


\title{
Topology optimization using a mixed formulation: \\ An alternative way to solve pressure load problems
}

\author{
O. Sigmund ${ }^{\mathrm{a}, *}$, P.M. Clausen ${ }^{\mathrm{a}, 1}$ \\ ${ }^{a}$ Department of Mechanical Engineering, Solid Mechanics, Technical University of \\ Denmark. Nils Koppels Alle, Building 404, DK-2800 Lyngby, Denmark
}

\begin{abstract}
A shortcoming of the traditional density based approach to topology optimization is the handling of design dependent loads that relate to boundary data, such as for example pressure loads. Previous works have introduced spline and iso-density curves or alternative parametrization schemes to determine the load surfaces. In this work we suggest a new way to solve pressure load problems in topology optimization. Using a mixed displacement-pressure formulation for the underlying finite element problem, we define the void phase to be an incompressible hydrostatic fluid. In this way we can transfer pressure loads through the fluid without any needs for special load surface parametrizations. The method is easily implemented in the standard density approach and is demonstrated to work efficiently for both $2 \mathrm{D}$ and 3D problems. By extending the method to a three phase (solid/fluid/void) design method we also demonstrate design of water containing dams.
\end{abstract}

Key words: Structural optimization, topology optimization, finite elements, mixed forms, incompressibility.

\footnotetext{
* Corresponding author.

Email addresses: sigmund@mek.dtu.dk (O. Sigmund), peter.clausen@fe-design.de (P.M. Clausen).

$U R L$ : www.topopt.dtu.dk (O. Sigmund).

1 Now FE-design GmbH, Karlsruhe, Germany
} 


\section{Introduction}

Since its introduction almost two decades ago [1], the topology optimization method for continuum structures [2] has increased tremendously in popularity and is now being used as an everyday mechanical design tool in larger industries and academia all over the world. Also a number of commercial topology optimization tools have been developed, either based on special Finite Element (FE) solvers or as add-ons to standard commercial FE packages.

While in academia, the applications of topology optimization methods has expanded to a multitude of problems in material design, MicroElectroMechanical Systems (MEMS), fluids, wave-propagation and nano-optics (see [2] for an overview), the most common design problems that are solved by commercial codes are still compliance minimization problems and maximization of lowest eigenfrequency problems with constraints on material resource. Although the solution procedures for these kinds of problems have matured to a satisfactory level, there are still a number of open or less-than-satisfactorily resolved issues with the fundamental topology optimization method. One group of problems concerns inclusion of manufacturing constraints such as minimum and maximum length-scale, draw directions and extrusion constraints; another group concerns inclusion of design dependent loads like pressure load problems.

In pressure load problems, the position of the loads depend on the shape and topology. Such problems are encountered in hydrostatics and dynamics of wind, water and snow loaded mechanical and civil structures such as ships, submerged structures, airplanes, pumps, etc.

A number of papers have addressed the pressure load problems in topology optimization. The Aalborg group [3-5] has suggested a formulation where the unknown load application curve is determined from an iso-density curve. The sensitivities of the distributed loads with respect to design changes are obtained from an efficient finite difference formulation and a scheme that prevents ill-defined load curves is suggested. In [6] it is suggested to implement the pressure loads by a manipulation of thermal prestrains and a special scheme for identification of "fluid" and void elements is applied. In [7] it is suggested to parametrize a load curve by a spline function and updating element densities and spline parameters in an integrated approach. A phase-field method involving a triple-well function that allows for distribution of three material phases (solid/fluid/void) is introduced in [8]. The scheme includes an explicit penalization of intermediate densities as well as perimeter. In all the above papers, it is stated that the pressure load problem is much more difficult to solve than standard (fixed) load problems. Different more or less elaborate schemes have to be applied in order to update the loading surface. Lately, several applications of the level set method to topology optimization have appeared. Since there exist implicitly given curves describing the boundaries for this method, it is fairly straight forward to implement pressure loads in the 
level set method $[9,10]$.

In this paper we introduce a new way to solve the pressure load problem based on a mixed displacement-pressure (incompressible) formulation but using the standard density approach to topology optimization. Thus, the scheme can be implemented in existing softwares based on the density approach. In fact, the only necessary changes to the code lies in the interpolation scheme, the boundary conditions and possibly the linear system solver.

The idea of the method is the following. Instead of defining the equilibrium equations in the typical FE displacement formulation, we define it in mixed form by including the pressure as a separate variable. This makes it possible to define the void phase in the topology optimization formulation as a hydrostatic incompressible fluid, thus allowing for transfer of pressure from the external boundary conditions to the structure - independent of its shape or topology. A potentially weak point of this idea is that internal "void" regions in the structures also become incompressible (fluid-filled) - a possibility the optimization algorithm may take advantage of by using the void regions as "incompressible cavities". This possibility, that may or may not be physically relevant for a particular problem, can be avoided by introducing an extra (compressible) void phase in the design problem and limiting the volume fraction of the fluid phase. The method thus becomes a 3-phase (solid/fluid/void) topology optimization scheme (see e.g. refs. [11-13] for previous work on 3-phase topology optimization). This computational scheme immediately applies to the 2 as well as 3 dimensional cases without further modifications. Also, the idea may be applied to design of water loaded structures like dams or water towers by introducing mass density and gravity loads on the fluid and structural phases.

The paper is organized as follows. In section 2 we formulate the physical model and compare the standard and mixed formulations for the solving of elastoand hydrostatic problems. In section 3 we formulate the topology optimization problem for 2 and 3 phase problems. In section 4 we demonstrate the method by considering various test problems, some of which are known from the literature. In section 5 we draw the conclusions and discuss strong and weak points of the method. 


\section{Physical model}

\subsection{Standard form}

We consider an elastic body $\Omega$ in equilibrium. The governing equations in strong form for this structure are

$$
\left.\begin{array}{lc}
\sigma_{i j, j}+F_{i}=0, & \text { in } \Omega, \\
u_{i}=u_{i}^{*} & \text { on } \Gamma_{u}, \\
\sigma_{i j} n_{j}=T_{i} & \text { on } \Gamma_{T},
\end{array}\right\}
$$

where $\sigma_{i j}$ is the symmetric stress tensor, $F_{i}$ the volume forces, $u_{i}$ the displacement components, $n_{i}$ the surface normal, $u_{i}^{*}$ and $T_{i}$ are the prescribed displacement and traction forces, respectively, and $\Gamma_{u}$ and $\Gamma_{T}$ denote the parts of the boundaries of $\Omega$ that are controlled by displacement or traction boundary conditions, respectively.

In weak form, the equilibrium conditions (1) can be written as

$$
\int_{\Omega} \delta \varepsilon_{i j} \sigma_{i j} d \Omega-\int_{\Omega} \delta u_{i} F_{i} d \Gamma-\int_{\Gamma_{T}} \delta u_{i} T_{i} d \Gamma=0
$$

which has to hold for all kinematically admissible displacement variations $\delta u_{i}$ and associated strain variations $\delta \varepsilon_{i j}$. The strain tensor is defined as $\varepsilon_{i j}=$ $\frac{1}{2}\left(u_{i, j}+u_{j, i}\right)$ and indices run over the dimension.

Considering linear isotropic elasticity the stress tensor in terms of bulk modulus $K$ and shear modulus $G$ depends on the dimension

$$
\begin{aligned}
& \sigma_{i j}=\left(K-\frac{2}{3} G\right) \varepsilon_{k k} \delta_{i j}+2 G \varepsilon_{i j} \text { for } 3 \mathrm{D} \\
& \sigma_{i j}=(K-G) \varepsilon_{k k} \delta_{i j}+2 G \varepsilon_{i j} \text { for } 2 \mathrm{D}
\end{aligned}
$$

where $\delta_{i j}$ is Kronecker's delta.

In most standard topology optimization formulations, the constitutive law (3) is given in terms of the Young's modulus $E$ and the Poisson's ratio $\nu$. For reasons that will become clear later, we have here chosen to work with the bulk and shear moduli. The value of the shear modulus in terms of $E$ and $\nu$ is independent on dimension, $G=\frac{E}{2(1+\nu)}$, whereas the bulk modulus $K$ depends on dimension and strain assumption. For 3D, 2D plane strain and 2D plane stress the bulk modulus is given as $K=\frac{E}{3(1-2 \nu)}, K=\frac{E}{2(1+\nu)(1-2 \nu)}$ and $K=\frac{E}{2(1-\nu)}$, respectively. 


\subsection{Mixed form}

As an alternative to the pure displacement formulation discussed above, one may state the equilibrium problem in mixed form by introducing a pressure variable [14]

$$
p=-K \varepsilon_{k k}
$$

Inserting (4) in (3) we get the constitutive law in mixed form as

$$
\sigma_{i j}=2 G e_{i j}-\delta_{i j} p
$$

where $e_{i j}$ is the deviatoric strain tensor which depends on dimension

$$
\begin{aligned}
& e_{i j}=\varepsilon_{i j}-\frac{1}{3} \delta_{i j} \varepsilon_{k k} \text { for } 3 \mathrm{D} \\
& e_{i j}=\varepsilon_{i j}-\frac{1}{2} \delta_{i j} \varepsilon_{k k} \text { for } 2 \mathrm{D}
\end{aligned}
$$

In weak form, the equilibrium conditions for the mixed formulation can be written as

$$
\int_{\Omega} \delta \varepsilon_{i j} 2 G e_{i j} d \Omega-\int_{\Omega} \delta \varepsilon_{i j} \delta_{i j} p d \Omega-\int_{\Omega} \delta u_{i} F_{i} d \Omega-\int_{\Gamma_{T}} \delta u_{i} T_{i} d \Gamma=0
$$

and additionally, we have the weak form of (4)

$$
\int_{\Omega} \delta p\left(p / K+\varepsilon_{k k}\right) d \Omega=0
$$

Equations (7) and (8) have to hold for all kinematically admissible displacement variations $\delta u_{i}$ and pressure variations $\delta p$. 


\subsection{Material properties}

Depending on the choices of the bulk and shear moduli in (7)-(8), we can model different material behaviours

$$
\begin{aligned}
& K \text { finite, } G \text { finite } \rightarrow \text { normal (compressible) elasticity } \\
& K \gg G, G \text { finite } \rightarrow \text { (almost) incompressible elasticity } \\
& 1 / K=0, G \text { finite } \rightarrow \text { incompressible elasticity } \\
& K \text { finite, } G=0 \rightarrow \text { compressible inviscid hydrostatic fluid } \\
& 1 / K=0, G=0 \rightarrow \text { incompressible inviscid hydrostatic fluid } \\
& K \text { small, } G=0 \rightarrow \text { void (air) }
\end{aligned}
$$

We may assign different material types (or properties) to different subregions of the modelling domain $\Omega$. In this way, we may model normal elasticity problems as well as pressure load and coupled fluid-structure problems by the mixed formulation - all with the same equations and requiring no special considerations for the boundary interface conditions between the fluid and solid regions. This idea will be demonstrated in detail in the example section.

Depending on the material properties (9), the external boundary conditions for the displacements $u_{i}$ and pressure $p$ fields have to be defined appropriately. The boundary conditions for various scenarios will also be discussed in the example section.

From a topology optimization perspective (9) provides the ideal basis for setting up an interpolation scheme that relates local material properties to the design variables. Since there are only two different and independent material properties, it is straight forward to set up a SIMP scheme for 2 or 3-phase design as will be seen in section 3 .

\subsection{Finite element implementation}

For the finite element implementation of the equilibrium equations, we consider independent approximations for the displacements, pressures and design variables (discussed later). The shape functions for these three fields are denoted $\mathbf{N}_{u}, \mathbf{N}_{p}$ and $\mathbf{N}_{\mu}$, respectively. In order to describe different combinations of shape functions for the different fields, we use the following (standard) notation. For example, a 4-node quadrilateral element with bi-linear displacement interpolation and piece-wise constant pressure and design variable interpolation is called a Q-4/1/1 element and a triangular 6-node element with quadratic displacement, linear pressure and constant design variable interpo- 
lations is called a T-6/3/1 element (c.f. ref. [14]).

After discretization of the weak forms (7)-(8), the mixed form linear system to be solved has the format

$$
\left[\begin{array}{ll}
\mathbf{K} & \mathrm{C} \\
\mathrm{C}^{T} & \mathbf{D}
\end{array}\right]\left\{\begin{array}{l}
\mathbf{u} \\
\mathbf{p}
\end{array}\right\}=\left\{\begin{array}{l}
\mathbf{f} \\
\mathbf{0}
\end{array}\right\}
$$

where $\mathbf{K}$ is the discretized stiffness matrix corresponding to the first term of (7), $\mathbf{C}$ is a coupling matrix corresponding to the second term of (7) or (8), $\mathbf{D}$ corresponds to the first term of (8) and $\mathbf{f}$ is the load vector corresponding to the two last terms of (7) (for more details, the reader is referred to appendix A or $[14])$.

Both the standard and the mixed formulations have been implemented and verified in the commercial finite element package COMSOL (By Comsol A/B, Stockholm, Sweden). This FE-package can be called from Matlab scripts and allows for the testing of various shape functions for the three individual fields. Since the mixed formulation (and the associated topology optimization scheme) is prone to numerical instabilities such as checkerboard patterns (for the element-wise constant density or pressure interpolations) and "saddle form instabilities" (for the continuous density or pressure interpolations, c.f. [15]) it is important to choose the right interpolation schemes for the elements. In this work, we have found that Q-4/1/1 and Q-4/1/4 elements for the $2 \mathrm{D}$ examples and Q-8/1/1 and Q-8/1/8 for the 3D examples work well when combined with the sensitivity filter [16]. For the mixed displacement-pressure formulations, these elements are known only to be partly stable, however, based on our experience, this does in general not cause problems for our optimization scheme. In all the examples we have run, we did not experience any pressure fluctuations for the chosen interpolation schemes. This may be attributed to the fact that the sensitivity information is independent on the pressure as shown in appendix A, hence the optimizer cannot "take advantage" of pressure fluctuations. In order to ensure full stability, one should use higher order displacement interpolations (e.g. Q-9/3/1 or Q-9/3/4). However, since no problems were experienced, these are not used here for CPU-time reasons. We tested a range of different interpolation schemes in order to check if they resulted in different optimized topologies. Since only small quantitative differences were observed, we decided only to use the Q-4/1/1 and Q-4/1/4 schemes discussed above. These two interpolations schemes are used randomly for the considered examples. 


\section{The topology optimization problem}

The goal of a standard topology optimization procedure in linear elasticity [2] is to find the distribution of solid material that minimizes the compliance of a structure. This goal is obtained by introducing a continuous (density) design variable for each element in the structure, assigning an interpolation function that relates the element stiffnesses to the element design variables, performing sensitivity analyses and updating the variables by an optimality criteria algorithm or a math programming script in an iterative process.

Since so many papers have appeared on the topology optimization method by now, we will not go into further details about the algorithm and the formulation of the optimization problem but simply concentrate on the ideas of this paper that differ from the standard approach.

A standard way of relating element stiffness to the element design variable is the SIMP approach (Solid Isotropic Material with Penalization) [17-20]. Here, the Young's modulus of an element $E$ is defined as

$$
E(\mu)=\mu^{\eta} E_{0}
$$

where $E_{0}$ is the Young's modulus of solid material, $\mu$ is the element relative density and $\eta$ is a penalization parameter (usually $\eta=3$ is introduced to favor discrete solutions of the continuous problem [20]).

In order to ensure a positive definite stiffness matrix, the Young's modulus $E(\mu)$ in $(11)$ is not allowed to become zero. This can be avoided by introducing a non-zero lower bound on the design variable $\mu$ or by re-defining the SIMP scheme as

$$
E(\mu)=E_{v o i d}+\mu^{\eta}\left(E_{0}-E_{v o i d}\right)
$$

where $0<E_{\text {void }} \ll E_{0}$.

In the present formulation, the SIMP interpolation is based on the bulk and shear moduli instead of the Young's modulus. In this way, we can very simply interpolate between the different material behaviours indicated in (9). Denoting the material constants for elastic material $K_{0}$ and $G_{0}$, the material constants for (incompressible) fluid $K_{\text {fluid }}$ and $G_{\text {void }}$ and the material constants for air (void) as $K_{\text {void }}$ and $G_{\text {void }}$, we will consider the following 5 different scenarios:

(1) Standard elasticity problem ( $\mu=0$ (void), $\mu=1$ (elastic material))

$$
\left.\begin{array}{l}
K(\mu)=K_{\text {void }}+\mu^{\eta}\left(K_{0}-K_{\text {void }}\right) \\
G(\mu)=G_{\text {void }}+\mu^{\eta}\left(G_{0}-G_{\text {void }}\right)
\end{array}\right\}
$$


(2) Incompressible formulation ( $\mu=0$ (incompressible elastic material), $\mu=$ 1 (fluid))

$$
\left.\begin{array}{l}
K(\mu)=K_{\text {fluid }}=K_{0} \\
G(\mu)=G_{\text {void }}+\left(1-\mu^{\eta}\right)\left(G_{0}-G_{\text {void }}\right)
\end{array}\right\}
$$

(3) Incompressible material and void ( $\mu=0$ (void), $\mu=1$ (incompressible elastic material))

$$
\left.\begin{array}{l}
K(\mu)=K_{\text {void }}+\mu^{\eta}\left(K_{0}-K_{\text {void }}\right) \\
G(\mu)=G_{\text {void }}+\mu^{\eta}\left(G_{0}-G_{\text {void }}\right)
\end{array}\right\}
$$

(4) Compressible elastic material with fluid ( $\mu=0$ (fluid), $\mu=1$ (elastic material))

$$
\left.\begin{array}{l}
K(\mu)=\mu^{\eta} K_{0}+\left(1-\mu^{\eta}\right) K_{\text {fluid }} \\
G(\mu)=G_{\text {void }}+\left(1-\mu^{\eta}\right)\left(G_{0}-G_{\text {void }}\right)
\end{array}\right\}
$$

(5) Elastic material with both incompressible fluid and void ( $\mu_{1}=0$ (void), $\mu_{1}=1$ and $\mu_{2}=0$ (elastic material), $\mu_{1}=1$ and $\mu_{2}=1$ (fluid)). For later use, we will also add a mass density interpolation for this scenario.

$$
\left.\begin{array}{l}
K\left(\mu_{1}, \mu_{2}\right)=K_{\text {void }}+\mu_{1}^{\eta}\left[\mu_{2}^{\eta} K_{\text {fluid }}+\left(1-\mu_{2}^{\eta}\right) K_{0}-K_{\text {void }}\right] \\
G\left(\mu_{1}, \mu_{2}\right)=G_{\text {void }}+\mu_{1}^{\eta}\left(1-\mu_{2}^{\eta}\right)\left(G_{0}-G_{\text {void }}\right) \\
\rho\left(\mu_{1}, \mu_{2}\right)=\rho_{\text {void }}+\mu_{1}\left[\mu_{2} \rho_{\text {fluid }}+\left(1-\mu_{2}\right) \rho_{0}-\rho_{\text {void }}\right]
\end{array}\right\}
$$

In the above cases we have perfect incompressibility of the fluid if $K_{\text {fluid }}=\infty$ or alternatively $K_{\text {fluid }} \gg G_{0}$ results in almost incompressibility ${ }^{2}$.

Note that case 4 above (fluid and compressible material) corresponds to case 2 (fluid and incompressible material) if one inserts $K_{0}=K_{\text {fluid }}$ in case 4 .

Case 5 above is a 3 -phase design problem (solid/fluid/void) and includes 2 design variables $\mu_{1}$ and $\mu_{2}$. The first variable determines whether there is matter in the element or not; the second variable determines whether the matter is fluid or an elastic solid. In fact, cases 1-4 above may be considered as special cases of case 5 . Case 1 corresponds to case 5 with $\mu_{2}=1$, case 2

$\overline{2}$ In practice it is not necessary to use a very large fluid bulk modulus. The example section will demonstrate that the resulting topologies do not change for fluid bulk moduli of 10-20 times the elastic shear modulus and above (e.g. $K_{\text {fluid }}="{ }^{\prime \prime} \geq$ $\left.20 G_{0}\right)$. 
corresponds to case 5 with $\mu_{1}=1$ (and $K_{0}=K_{\text {fluid }}$ ), case 3 corresponds to case 5 with $\mu_{2}=0$ and case 4 corresponds to case 5 with $\mu_{1}=1$.

\subsection{Interpolation with the RAMP formulation}

The RAMP (Rational Approximations of Material Properties) scheme [21] was proposed as an alternative to the SIMP interpolation scheme discussed above. The primal idea of the RAMP scheme is to obtain a concave design problem, thus ensuring convergence to pure $0 / 1$ (black and white) solutions (for compliance problems). However, as pointed out by [22] and in other connections, the scheme also has the advantage that the derivative for small density values is non-zero as opposed to the SIMP scheme. This means that problems with spurious modes for eigenvalue problems with low stiffness to weight ratios in low density regions are eliminated (see [23] for a modification of the SIMP scheme that also eliminates this problem). Likewise, [22] proposes to eliminate convergence problems in self-weight problems by the RAMP approach combined with a modified optimization scheme based on the Method of Moving Asymptotes [24]. Here, we also apply RAMP in connection with selfweight, c.f. section 4.5 .

The standard two-phase RAMP interpolation scheme is

$$
E(\mu)=E_{v o i d}+\frac{\mu\left(E_{0}-E_{v o i d}\right)}{1+q(1-\mu)}
$$

where $q \geq 0$ is a penalization factor.

For the three phase problem considered (17), the RAMP interpolation becomes

$$
\left.\begin{array}{l}
K\left(\mu_{1}, \mu_{2}\right)=K_{\text {void }}+\frac{\mu_{1}}{1+q\left(1-\mu_{1}\right)}\left[K_{0}-K_{\text {void }}+\frac{\mu_{2}}{1+q\left(1-\mu_{2}\right)}\left(K_{\text {fluid }}-K_{0}\right)\right] \\
G\left(\mu_{1}, \mu_{2}\right)=G_{\text {void }}+\frac{\mu_{1}\left(1-\mu_{2}\right)}{\left(1+q\left(1-\mu_{1}\right)\right)\left(1+q \mu_{2}\right)}\left(G_{0}-G_{\text {void }}\right) \\
\rho\left(\mu_{1}, \mu_{2}\right)=\rho_{\text {void }}+\mu_{1}\left[\rho_{0}-\rho_{\text {void }}+\mu_{2}\left(\rho_{\text {fluid }}-\rho_{0}\right)\right]
\end{array}\right\}
$$

\subsection{Objective function and optimization problem}

In standard compliance optimization problems, the compliance may be calculated as the integral of the strain energy density over the total volume of the design domain which equals the work done by the external forces. With the present incompressible formulation, the compliance may be calculated in exactly the same way. Note that since the fluid domain is incompressible and has negligible shear stiffness the strain energy stored in the fluid domain will be diminutive compared to the elastic domain. Therefore, we can also here apply the usual energy objective function. 
The expression for the energy is

$$
W=\frac{1}{2} \int_{\Omega} \sigma_{i j} \varepsilon_{i j} d \Omega
$$

Including volume fraction constraints on material and fluid, the full optimization problem may then be formulated as

$$
\left.\begin{array}{rl}
\min _{\mu_{1}, \mu_{2}} & : W\left(\mu_{1}, \mu_{2}\right) \\
\text { s.t. } & : \text { Equilibrium }(7) \text { and }(8) \\
& : \int_{\Omega} \mu_{1}\left(1-\mu_{2}\right) d \Omega / \int_{\Omega} d \Omega \leq f_{0} \\
& : \int_{\Omega} \mu_{1} \mu_{2} d \Omega / \int_{\Omega} d \Omega \geq f_{\text {fluid }} \\
& : 0 \leq \mu_{1} \leq 1, \quad 0 \leq \mu_{2} \leq 1
\end{array}\right\}
$$

where $f_{0}$ and $f_{\text {fluid }}$ are the volume fractions for elastic material and fluid, respectively.

\section{$3.3 \quad$ Numerical implementation}

The topology optimization problem (21) is solved using Matlab scripts calling the FE-package COMSOL. As demonstrated in [25] the Matlab script can be formulated very compactly using COMSOL calls and the derivation of sensitivity information can be performed (semi-)automatically. This is obtained by defining the design variable fields $\mu_{1}$ and $\mu_{2}$ as extra fields in the FE model. The solver only solves for the original displacement and pressure fields. For the sensitivity analysis COMSOL's built-in automatic differentiation sets up the adjoint equations automatically (if needed) and the sensitivity information is found from the residual expressions for the design variable fields (see [25] for more details). Having defined the design variables as separate fields makes it possible to interpolate the design fields using all COMSOL's built-in element interpolation schemes. This makes is possible to test different element interpolation schemes and objective functions by changing a single line in the Matlab script. For completeness, however, we have added appendix A that demonstrates the analytical derivation of the sensitivities of the strain energy objective function.

Since the emphasis in this paper is on the physical concept and not the implementation, no further details about the implementation will be given here. However, it is noted that the automatically calculated sensitivities have been confirmed by finite differences checks. The optimization problems is solved by the Method of Moving Asymptotes (MMA, [24]). Finally, as with all other 


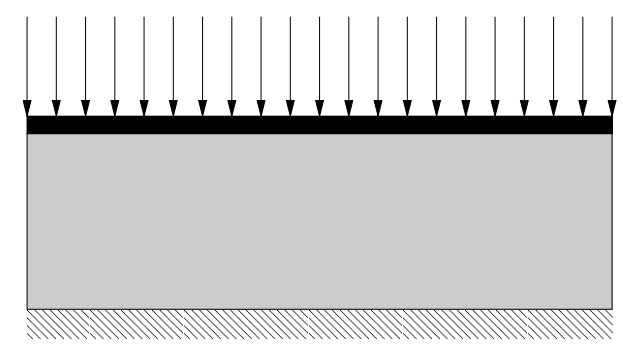

Fig. 1. Design domain for bridge example.

density formulations, the proposed topology optimization scheme is prone to numerical problems like checkerboards and mesh-dependencies [26]. In order to avoid this problem we use the mesh-independency filtering scheme described in ref. [16].

\section{Examples}

This section includes a number of examples intended to demonstrate the idea and efficiency of the method. In the examples, we consider non-dimensionalized properties and dimensions for simplicity, however, the method works just as well for real physical values. In all figures, black denotes solid elastic material and white may denote fluid or void regions depending on the example. In the three-phase cases where confusion is possible, the fluid regions are crosshatched.

\subsection{Bridge}

The design domain for the first test example is shown in Fig. 1: a rectangular design domain of dimension 1 by $1 / 3$, the top $1 / 10$ of the domain is fixed to be solid and the structure is subjected to a distributed vertical unit traction load on the top-surface. Unless otherwise noted, the design domain is discretized by 1080 square Q-4/1/1 elements. The volume fraction of solid material is $f_{0}=0.3$, the penalization power for the SIMP interpolation is $p=3$ and the filter size is 1.5 times the element size.

The optimized topologies for the standard displacement approach and the mixed form are seen in Fig. 2. For this case the material properties for the solid material are $E=1$ and $\nu=0.3$, resulting in the bulk and shear moduli $K_{0}=1 / 1.4$ and $G_{0}=1 / 2.6$ for the plane stress assumption and $K_{\text {void }}=$ $G_{\text {void }}=10^{-6}$ and we use the interpolation scheme (13). It is seen that the two designs are hard to distinguish, confirming that the mixed formulation for normal compressible materials produces similar results as for the pure displacement formulation. The small difference is due to the difference in field interpolations between the two schemes. 

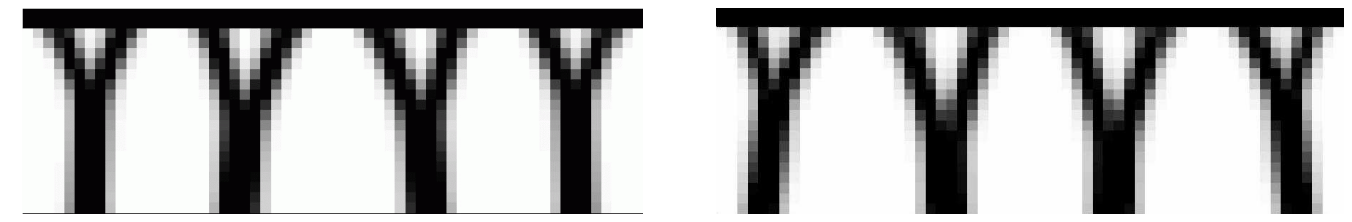

Fig. 2. Optimized bridge topologies for standard compressible elasticity. Left: standard displacement based approach. Right: mixed form approach. The initial and final compliances are $W_{\text {init }}=5.244$ and $W_{\text {final }}=0.788$ and $W_{\text {init }}=5.245$ and $W_{\text {final }}=0.806$, for the two cases, respectively.
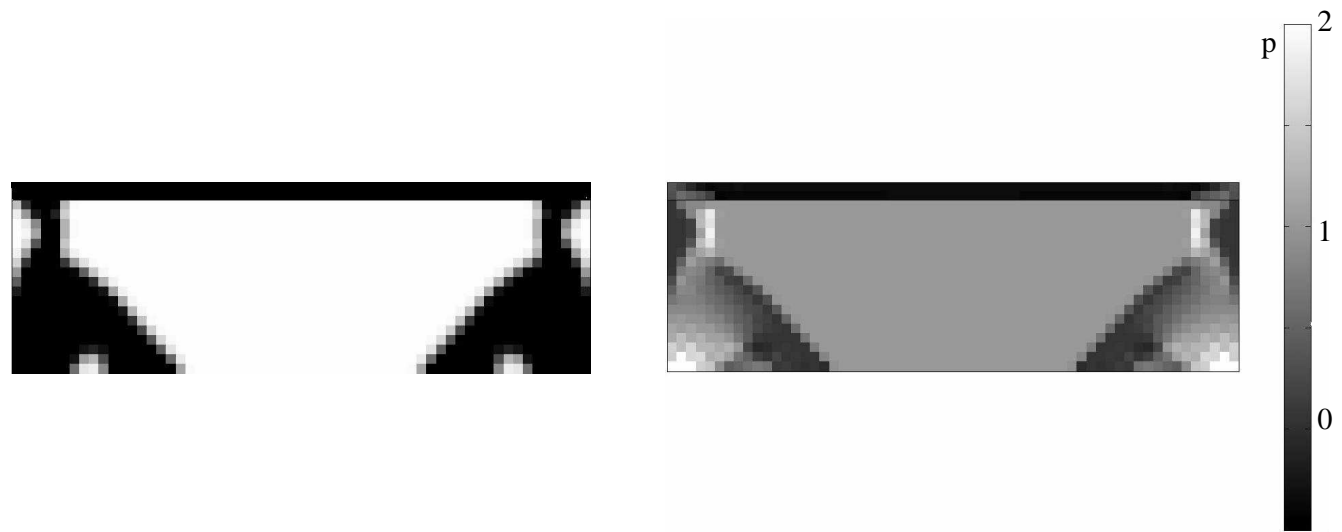

Fig. 3. Optimized bridge topology for the incompressible case and $K_{0}=K_{\text {fluid }}=10^{6}$. Left: material distribution. Right: pressure field. The compliance is $W_{\text {final }}=0.181$.

Next, we optimize using the incompressible formulation (14). We try out three cases: $K_{0}=K_{\text {fluid }}=\infty, K_{0}=K_{\text {fluid }}=10^{6}$ and $K_{0}=K_{\text {fluid }}=10$ in order to check whether the "enforcement of incompressibility" is critical for the optimal design. The shear modulus for the solid phase remains $G_{0}=1 / 2.6$ as before. The optimized topology for the case $K_{0}=K_{\text {fluid }}=10^{6}$ is shown in Fig. 3, left. The topologies obtained for $K_{0}=K_{\text {fluid }}=\infty$ and $K_{0}=K_{\text {fluid }}=10$ are indistinguishable from the first topology. However, the topology is seen to be significantly different from the topologies obtained for the compressible formulation in Fig. 2. The explanation is that the internal (white) part in Fig. 3(left) can be seen as a (incompressible) fluid-filled cavity that contributes to the overall stiffness of the structure. The main purpose of the solid regions are thus to encapsulate the fluid efficiently. The compliance of the optimized topology is $W_{\text {final }}=0.181$. If the cavity topology is modelled using standard compressible modelling, the compliance is $W_{\text {comp }}=170.4$, i.e. it is extremely inefficient if the cavity does not provide stiffness. In contrast, the compliance of the topology obtained for the compressible formulation (Fig. 2) is $W=0.339$ when modelled using the incompressible formulation, i.e. close to two times worse than the incompressible cavity topology.

In Fig. 3(right) we show the pressure field for the cavity topology. It is seen that the pressure is equal to 1 in the cavity - corresponding to the externally 

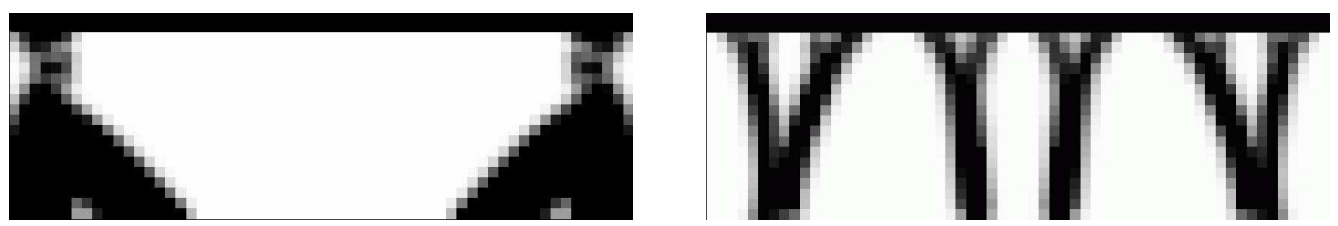

Fig. 4. Optimized bridge topology for compressible elastic material and fluid (left) and incompressible material and void (right). The compliance for the two cases are $W_{\text {final }}=0.240$ and $W_{\text {final }}=0.546$.

applied pressure load.

The latter result demonstrates the idea of the method. Incompressible fluid domains may transmit surface loads to the topology, thus avoiding the need for identifying the pressure surface as is the case for other formulations. The idea will be discussed and further demonstrated in the following examples.

Before proceeding, however, it should be noted that the solid material present in the cavity example (Fig. 3) corresponds to incompressible material, e.g. rubber. If we want to design problems with normal compressible elastic material and fluid inclusions, we have to use the interpolation scheme (16) as described in section 3. For this case we select $K_{0}=1 / 1.4$ and $K_{\text {fluid }}=10$. Fig. 4(left) shows the optimized topology for this case. The topology is seen to be only slightly different from the purely incompressible case in Fig. 3.

The last application of the bridge problem is the interpolation scheme (15) for void and incompressible material. Numerically, this example is harder to make converge towards a discrete (black and white) topology. The reason is that the optimization scheme tries to take advantage of the (partial) incompressibility of grey regions in order to get minimum compliance. The problem can be avoided by selecting not too big values of the solid bulk modulus (i.e. $K_{0}$ not bigger than 10-20 times the shear modulus) and a large penalty exponent (e.g. $\eta=5$ ). The resulting topology is seen in Fig. 4(right) and is seen to be quite similar to the previous cases with void inclusions. In order to study the possible differences in optimized topologies for compressible and incompressible materials one would have to run other test cases with finer meshes and varying geometries and load cases. However, since the emphasis in this paper is on the pressure transmitting capabilities of the mixed formulation and not on the topology optimization with incompressible elastic materials, those studies will be presented elsewhere.

\subsection{Internally pressurized lid}

This example has been used in the literature to demonstrate schemes for pressure load problems $[3-6,10]$. The design domain with indication of boundary conditions corresponding to a simply supported stubby beam of length 1 and height $1 / 2$ is shown in Fig. 5a. The pressure load may for the mixed formu- 


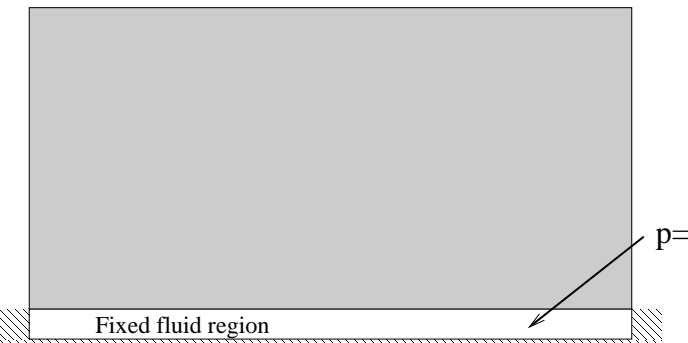

a)

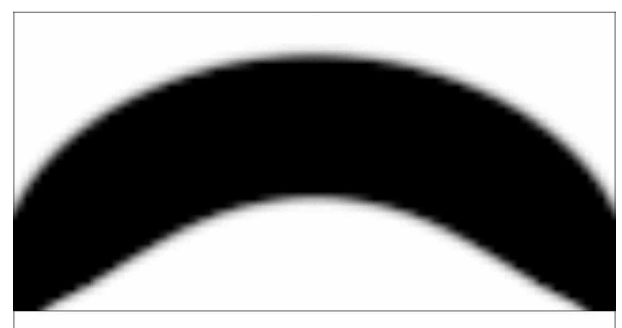

c)

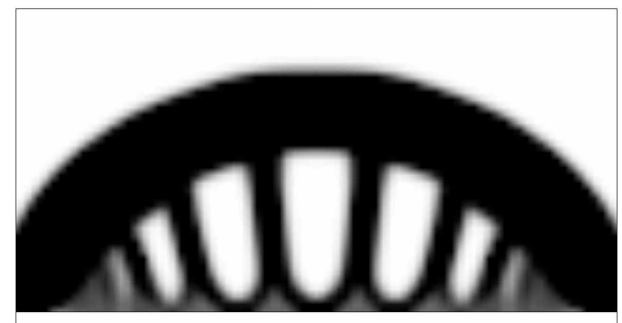

b)

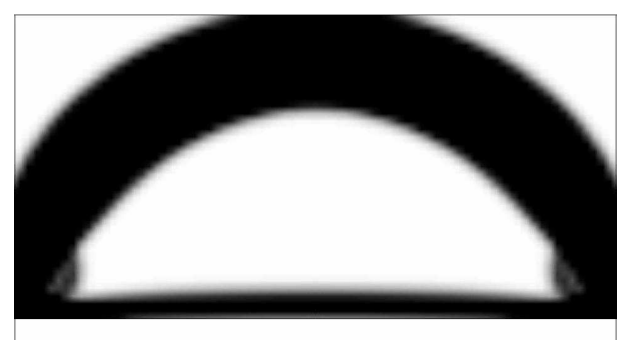

d)

Fig. 5. a) Design domain and boundary conditions for internally loaded lid. The white region below the design domain indicates a region fixed to be fluid. b) Reference case with optimized topology for pressure load fixed to the lower edge of the design domain. c) Optimized topology with pressure load. d) Erroneously optimized topology using quadratic displacement interpolation and point supports (see comments in text).

lation be introduced by different boundary conditions. Common for all cases, however, is that a narrow fixed fluid region is introduced below the design domain. The fluid region is put under pressure either by removing the supports on the lower edge and applying a vertically distributed traction load, by prescribing the vertical displacements of the lower edge or by prescribing the pressure on the left, right and lower edges of the fixed fluid region. For the following cases we used the latter approach, however, it has been tested that the other two formulations result in essentially the same responses and topologies. For this case, we again use rectangular elements with bi-linear displacement interpolation and element-wise constant pressure interpolation but for the design field we use a bi-linear interpolation, i.e. Q- $4 / 1 / 4^{3}$. In the following examples only half the design domain is discretized and there are 1681 nodal design variables. A volume fraction of $f_{0}=0.5$ may be filled with solid material with $E=1$ and $\nu=0.3$. The mesh-independency filter size is again equal to 1.5 times the element size.

As a reference we first optimize the structure for fixed unit tractions on the lower edge of the design domain and non-fluid void regions (i.e. interpolation scheme (13)). The optimized topology is seen to be the well-known bridge-like

$\overline{3}$ Piece-wise constant and bi-linear interpolations for the design field seem to work equally well. In the examples we switch between the two for demonstrative purposes. 
solution (Fig. 5b) which has a compliance of $W_{\text {final }}=1.12$. As a check we can also analyze this optimized structure with incompressible void phase (16) and with boundary conditions corresponding to prescribed $p=1$ on the lower, left and right boundaries of the fixed fluid region. Also in this case the compliance is $W=1.12$, confirming that the idea of transmitting surface loads through an incompressible fluid gives a correct representation.

Next we optimized the structure using interpolation scheme (16) with $K_{\text {fluid }}=$ 100. The optimized topology is seen in Fig. 5c and is seen to be an arch-like structure known from the previous works on pressure load problems. The final compliance of the arch-like structure is $W_{\text {final }}=0.94$, i.e. $16 \%$ better than for the fixed traction problem. This result corresponds well with the $17.67 \%$ improvement reported in Ref. [4] which was obtained for a coarser discretization (400 elements) and an iso-density curve for imposing the pressure load surface.

As a curiosity we also show the optimized topology (Fig. 5d) for the case where we use quadratic displacement interpolation, bi-linear pressure and density for the finite element interpolation (i.e. Q-8/4/4). The resulting structure is seen to have a solid bar at the bottom of the design domain. A first reaction to this topological difference is to assume that it is a local minimum. However, this is not the case. Topology d) is better than topology c) for this case with higher order displacement interpolation. The explanation can be found in the boundary conditions. The (non-physical) point supports cause extremely big displacements in the support regions. By introducing a horizontal bar between the supports, these displacements are significantly reduced. If we change the point supports to distributed supports (extending 5\% into the design domain from both sides), we obtain the same topology as for the linear displacement interpolation case of Fig. 5c. The morale of this example is that point supports (and probably point loads) as often seen in topology optimization implementation, apart from giving inaccurate answers to the FE problem, also may cause erroneous optimized topologies. This was not unexpected but has to our knowledge seldomly been demonstrated in the literature.

\subsection{Externally pressurized lid}

The third example considers the optimization of an externally pressurized lid which is also known from the literature. The design domain is shown in Fig. 6a. The finite element interpolation for this example is Q-4/1/4 and there are 3111 nodal design variables (only considering half of the domain due to symmetry). Again we consider the plane stress assumption and the bulk modulus of the material to be distributed is $K_{0}=1 / 1.4$ whereas in the first case the fluid bulk modulus is $K_{\text {fluid }}=10$ and in the second case $K_{\text {fluid }}=100$, the volume fraction of solid material is $f_{0}=0.2$ and other properties correspond to the previous example. 


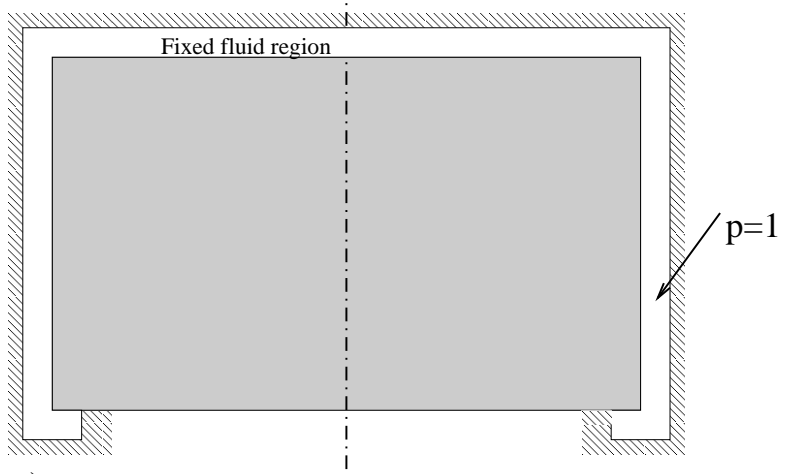

a)

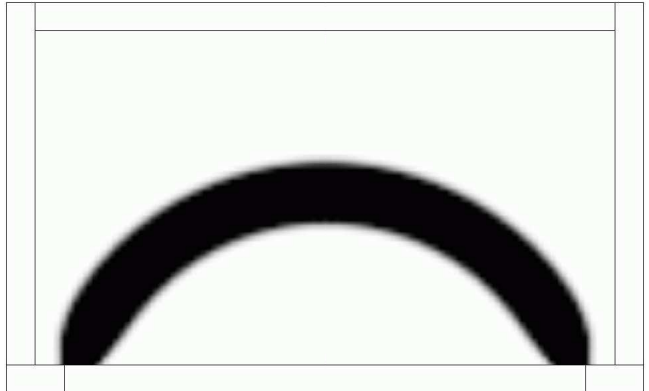

b)

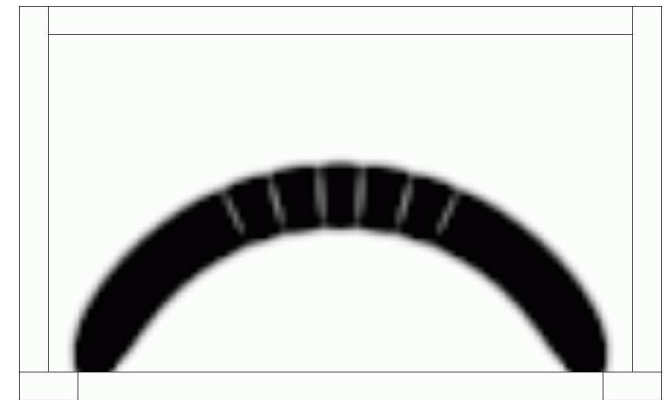

c)

Fig. 6. a) Design domain and boundary conditions for externally loaded lid. The white region around the design domain indicates a region fixed to be fluid (with pressure $p=1$ ). b) Optimized topology for $K_{0}=10$. c) Optimized topology for $K_{0}=100$.

The optimized topologies for the two cases are seen in Fig. $6 \mathrm{~b}$ and c. The initial compliance is for both cases $W_{\text {init }}=21.8$ and for the optimized topologies we obtained $W_{\text {final }}=0.76$ and $W_{\text {final }}=0.74$, respectively. As expected, the topology for the case of $K_{\text {fluid }}=10$ (Fig. 6b) is an almost circular arch that supports the pressure load efficiently. The number of iterations for this case is 80. For the case of $K_{\text {fluid }}=100$ (Fig. 6c), the convergence is less stable and 123 iterations are needed. Interestingly, we see that there are radially extending internal "cracks" in the upper part of the arch. An interpretation of these "cracks" is that the optimization takes advantage of the incompressibility of the void phase. The closed cracks have high compressive stiffness but low shear stiffness which is advantageous for the arch structure which is subject to purely tangential compressive stresses. For higher values of the fluid bulk modulus, convergence becomes unstable since the optimizer tries to make instable, "porous", intermediate density structures with high bulk modulus and finite shear modulus.

As noted earlier, the formation of the cracks and porous regions and the resulting somewhat unstable convergence may be avoided by choosing a bulk modulus of the liquid phase not too much bigger than for the solid phase (e.g. $K_{\text {fluid }}$ 10-20 times $K_{0}$ as in Fig. $6 \mathrm{~b}$ ). 


\subsection{Cylinder}

As a fourth example we consider the piston sketched in Figure 7a. This example is somewhat similar to the piston problem discussed in ref. [8]. The design domain is rectangular, supported by rollers on the vertical walls (cylinder walls), fixed at the center of the bottom edge and subjected to a pressure load on the top surface. Here, we use the three-phase interpolation scheme (17) in order to distribute compressible elastic material, incompressible fluid and void in the design domain. The constraint on elastic material is $f_{0}=0.3$. We consider $2 \mathrm{~d}$ plane strain assumption, $K_{\text {fluid }}=10, K_{\text {void }}=G_{\text {void }}=G_{\text {fluid }}=10^{-6}$, $K_{0}=1 / 1.4$ and $G_{0}=1 / 2.6$.

First we constrain the fluid volume fraction in the design domain to $f_{\text {fluid }}=$ 0 and get the results seen in Figure 7 e. The compliance is $W_{\text {final }}=8.94$ and we see that the optimized topology resembles a structure subjected to a fixed pressure load on the top surface of the design domain. If we allow for more fluid in the design domain $\left(f_{\text {fluid }}=0.05\right)$ we get the better topology seen in Figure $7 \mathrm{~d}\left(W_{\text {final }}=7.52\right)$. We note how the pressure load surface has changed to improve the response. If we further increase the allowed fluid volume fraction to 0.1 and 0.3 (Figures $7 \mathrm{c}$ and b, respectively), we note how the fluid also starts occupying the internal holes in the structure, giving rise to increased overall stiffnesses $\left(W_{\text {final }}=7.15\right.$ and $W_{\text {final }}=6.45$ for the two designs, respectively).

This example demonstrates how to eliminate a potential weak point of the method. If we had only used the two-phase (solid-fluid) approach to solve the piston example we would have obtained the result seen in Figure $7 \mathrm{~b}$, i.e. a structure that takes advantage of filling interior holes with (incompressible) fluid. This may be relevant in certain applications like dam design, however, it may also be undesirable in many other applications. The example demonstrates how we, using the three phase approach, can make sure that the interior parts are dry by constraining the volume fraction of the fluid. In practise, this must happen in an interactive process, where the fluid volume fraction is increased up to the point when fluid-filled cavities are first seen. Alternatively one could introduce "filling-detection" schemes as discussed in [6]. Both the volume constraint scheme suggested here and the "filling-detection" scheme which are introduced to control the fluid filled regions have their disadvantages and the best method probably still has to be found. The scheme suggested here requires a trial-and-error approach to come up with the right constraint on the fluid volume which, especially for $3 \mathrm{D}$, might be very time-consuming. The "filling-detection" requires a well defined jump between fluid and solid regions and requires careful tuning - especially for the dam examples presented later which have non well-defined solid material at the top of the structure.

An interesting observation that can be drawn from studying the optimized topologies in the examples shown so far is that structural parts subject to 


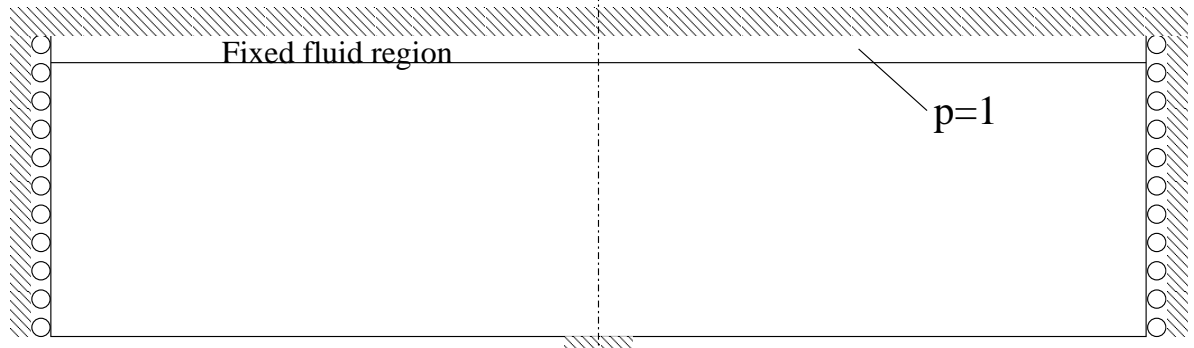

a)
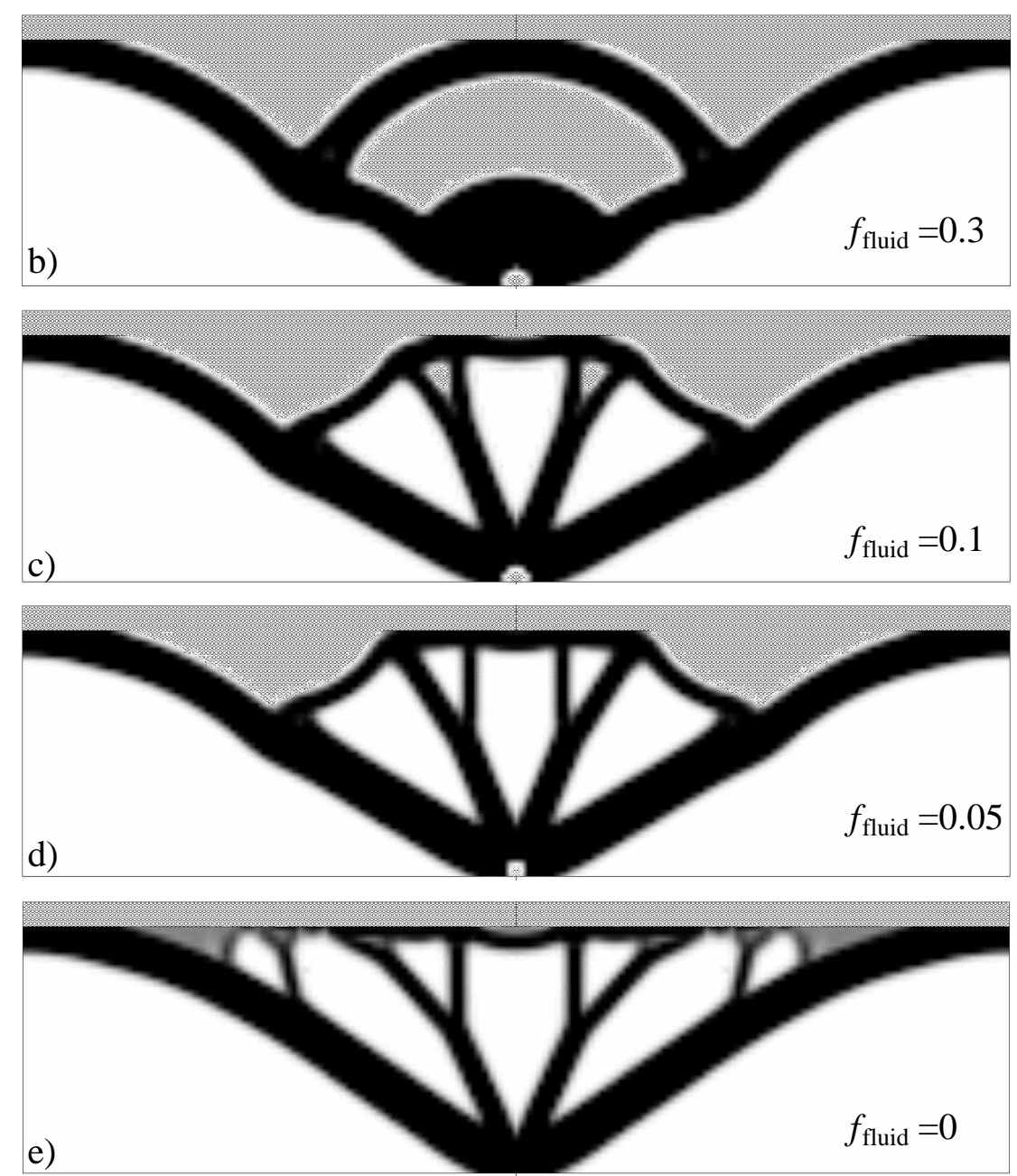

Fig. 7. Design of a piston with fluid volume fraction constraint. Grey areas denote fluid regions. a) Design domain, b-e) optimized topologies for $f_{\text {fluid }}=0.3$, $f_{\text {fluid }}=0.1, f_{\text {fluid }}=0.05$ and $f_{\text {fluid }}=0$, respectively.

pressure loads generally take arch shapes whereas other structural components are more or less straight. In this way, the optimization makes sure that all structural components always are in a state of compression or tension - never in bending. 


\subsection{Dam}

The suggested three phase RAMP interpolation scheme (19) allows for the design of dams subjected to pressure loads. In reality, the design of a dam is a very complex problem. Apart from the static loading caused by the water pressure, one should also consider earth-quake loadings, waves, elasticity of foundations, porosity, seepage, etc.. Here we consider a very simplified problem that only has static loading from the water. A similar problems has been solved in [7] with the loading surface determined by a spline interpolation and in [8], however, with non-physical vertical supports at the top of the design domain, enforced in order to hinder contact between the fluid and void zones.

Introducing a mass density $\rho_{\text {fluid }}$ for the fluid as well as a gravity load $\left(F_{2}=\right.$ $\left.-g \rho_{\text {fluid }}\right)$ in $(7)$, one obtains a linear pressure profile in the fluid region increasing with the depth of the water. This is illustrated in Fig. 8. A three-phase design (c.f. the RAMP interpolation (19)) consisting of a void $\left(\mu_{1}=\mu_{2}=0\right)$, a solid $\left(\mu_{1}=1\right.$ and $\left.\mu_{2}=0\right)$ and a fluid $\left(\mu_{1}=1\right.$ and $\left.\mu_{2}=1\right)$ region is shown in Fig. 8a. The structure is fixed at the lower edge and supported in the horizontal direction on the right edge. The calculated pressure distribution for a fluid mass density of $\rho_{\text {fluid }}=1$ and a material mass density of $\rho_{0}=0$ (ignoring self weight of the dam) is seen in Fig. $8 \mathrm{~b}$ and for fluid and material mass density of $\rho_{\text {fluid }}=\rho_{0}=1$ (taking self-weight of the dam into account) in Fig. 8c. In both cases, it is seen that the pressure profile in the fluid regions varies linearly downwards from the water surface as would be expected. In the solid regions the maximum value of the pressure is highest in the lower left corner of the dam where, especially for the second case, the bending moment from the water pressure combined with the self-weight of the dam causes the large pressure. The work of the external forces (gravity loads) for the two cases are $W=0.308$ and $W=0.330$, respectively, which compares favorably to the compliance for a solid square box, supported at the bottom and with a linearly distributed external load (i.e. the same geometry but without the void and fluid regions), which are $W=0.298$ and $W=0.319$, respectively.

After having observed that the proposed three-phase interpolation actually models the physical system correctly, we may proceed to the topology optimization formulation. For the design of the dam, we minimize the work of the external (gravity) forces

$$
W_{e x t}=\int_{\Omega} F_{i} u_{i} d \Omega=\int_{\Omega} g \rho\left(\mu_{1}, \mu_{2}\right) u_{2} d \Omega,
$$

where the gravity constant is set to unity $g=1$.

We use the following data for the design problem: plane strain assumption, incompressible elastic material with $K_{0}=10, K_{\text {fluid }}=100$, linear density interpolation with 4141 nodal design variables, 4800 Q-4/1/4 elements, RAMP 


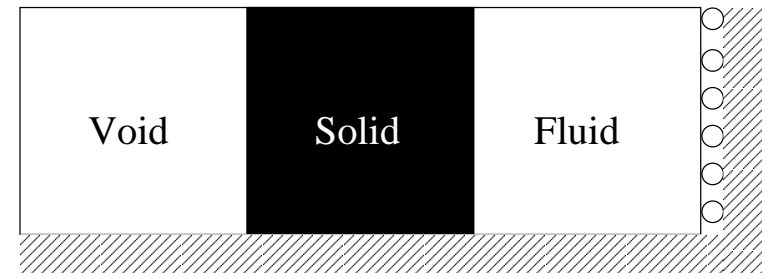

a)

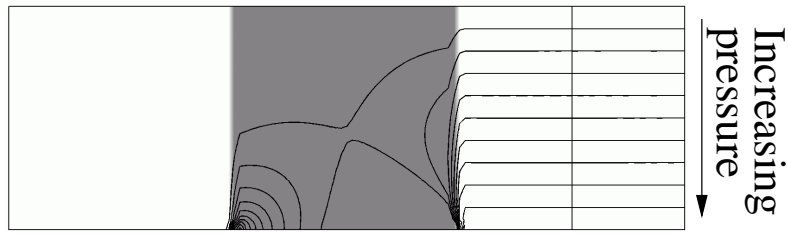

b)

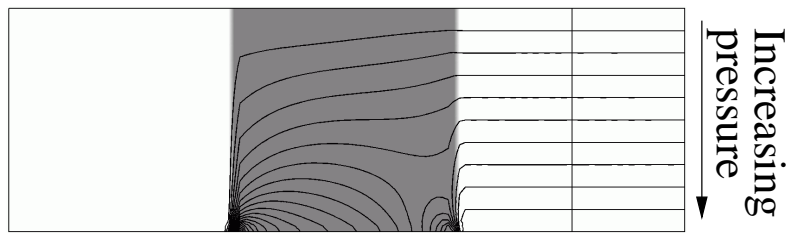

c)

Fig. 8. a) Structure and boundary conditions for demonstration of pressure load. b) Pressure level curves for $\rho_{\text {fluid }}=1$ and $\rho_{0}=0$ and c) pressure level curves for $\rho_{\text {fluid }}=\rho_{0}=1$.

penalization factor $q=5$ (c.f. (19)) and volume fraction constraint on the elastic material $f_{0}=0.4$ or $f_{0}=0.2$.

As a reference for the improvements obtained by the topology optimization, we perform a small shape parameter study. Intuitively, we expect the dam crosssectional shape to be a triangular structure. In Fig. 9 we plot the external work for varying shapes of the triangle for three different assumptions of the selfweight of the dam. We see that the optimal triangular dam shape varies with the self-weight. The higher the self weight, the more the dam "leans" towards the water. The reason for this is that the self-weight of the dam is used to counteract the pressure from the water or oppositely, the water pressure helps to carry the weight of the dam. The shape optimal dams are seen in the figure and the external works are $W=0.112, W=0.124$ and $W=0.226$ for the mass densities of the elastic material being $\rho_{0}=0, \rho_{0}=1$ and $\rho_{0}=2.3$ (the latter corresponding to the mass density of concrete), respectively. ${ }^{4}$

For the topology optimization, the design domain for the dam problem is shown in Fig. 10a. In order to ensure non-trivial solutions, we fix the right part of the design domain to be fluid. The starting guess for the optimiza-

4 Note that the shape study is performed for a dam made of incompressible elastic material. If the same study is performed for compressible elastic material, the resulting triangle shapes will be oriented slightly more towards the water (to the right). 


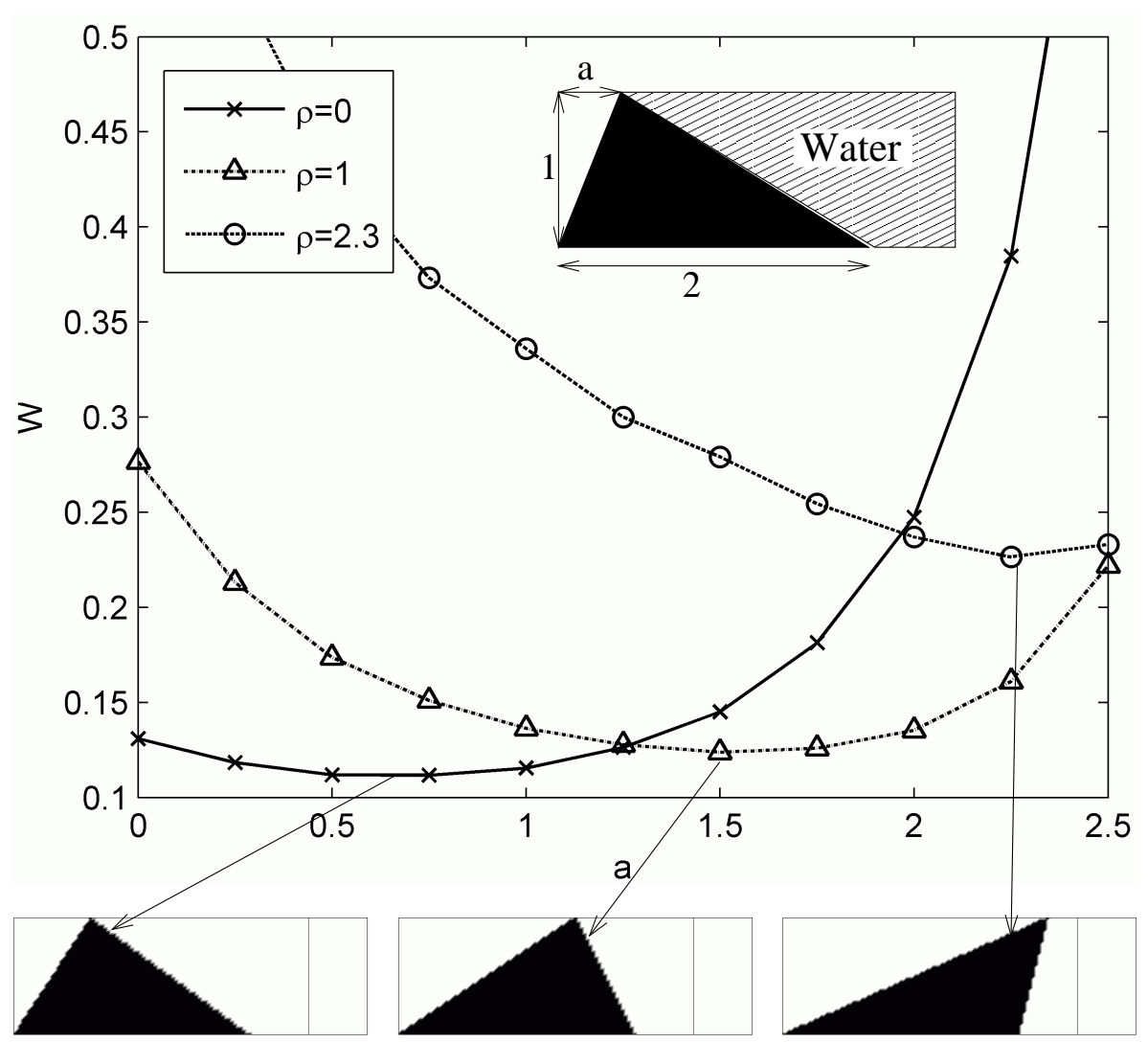

Fig. 9. Shape parameter study for dam example. The graph shows the work of external forces as function of the horizontal position of the upper corner of the triangle and the figures below show the optimal dam triangular dam shaped for the case of dam mass density $\rho_{0}=0,1$ and 2.3 , respectively.

tion is shown in Fig. 10b. The optimized topologies for mass density of the dam material of $\rho_{0}=0, \rho_{0}=1$ and $\rho_{0}=2.3$ are seen in Figs. 10c-e. The final values of the objective function are $W_{\text {final }}=0.116, W_{\text {final }}=0.125$ and $W_{\text {final }}=0.173$, respectively, which is $3 \%, 4 \%$ and $23 \%$ less than for the pure shape based designs in Fig. 9. For this choice of volume fraction the obtained topologies have practically no internal holes and could have been obtained by a shape optimization approach. In this connection it should be noted that the optimized topology for zero self-weight $\left(\rho_{0}=0\right)$ of the dam (Fig 10c) should correspond to the example from [7]. However, the obtained dam shapes are widely different. The reason for this discrepancy has yet to be explained but could be due to errors or limitations of the spline parametrization used in ref. [7]. In the present study, the results have been checked with two different commercial FE solvers.

In order to obtain some more interesting topologies for the dam, we reduce the available material amount to $f_{0}=0.2$. In this way we expect that the dams will become non-solid with internal holes. The results of this study are seen in Figs. 10f-h. In this case the final values of the objective function are $W_{\text {final }}=0.215, W_{\text {final }}=0.225$ and $W_{\text {final }}=0.251$ for the three different self- 


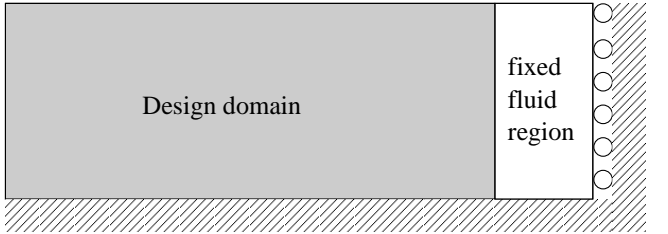

a)

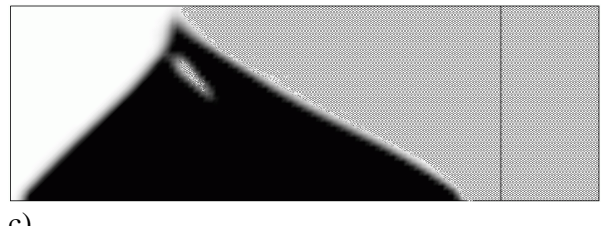

c)

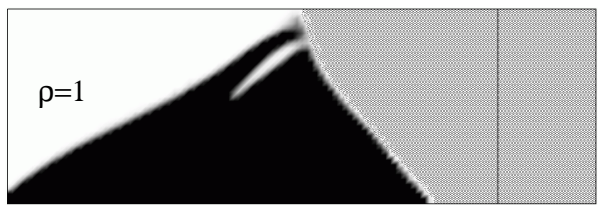

d)

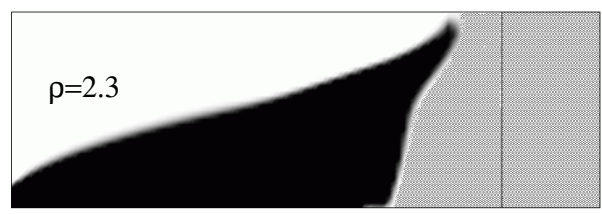

e)

$\mathrm{V}_{0}=0.4$

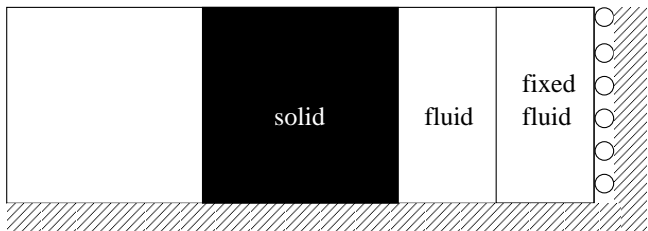

b)
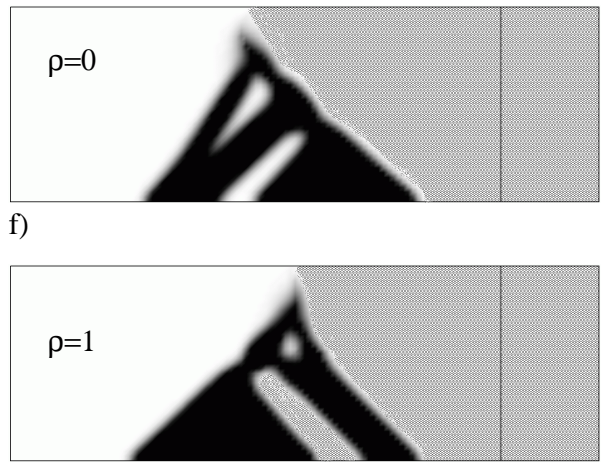

g)

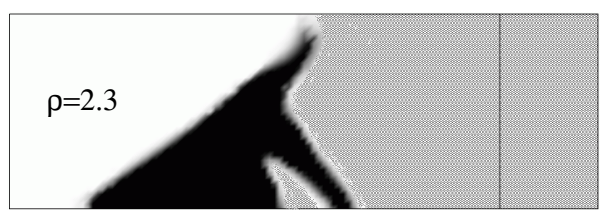

h)

$\mathrm{V}_{0}=0.2$

Fig. 10. a) Design domain and b) starting guess for dam example. c-e) Optimized topologies for material volume fraction $f_{0}=0.4$ and material density 0,1 and 2.3, respectively. f-h) Optimized topologies for material volume fraction $f_{0}=0.2$ and material density 0,1 and 2.3 , respectively.

weight cases. It is interesting to note that in Fig. $10 \mathrm{~g}$ the lower hole is fluid filled whereas the upper smaller hole is void (compressible). The optimization may take advantage of using internal incompressible cavities - an option that is also known from dam designs in reality.

Comparing the topologies optimized for different self weights reveals that the work of the external forces does not increase significantly and in fact for one case decreases when the self weight is increasing. This may sound contradictory, however, it may attributed to the optimizer producing topologies that counteract the (near) horizontal loads coming from the water pressure and the vertical gravity loads on the dam structure itself.

As noted in the beginning of this section, the presented dam examples are purely academic. In reality, many other loads would be acting on the structure and certainly the thickness of the dam would not go to zero at the top of the dam as in the presented examples. Rather they should have a finite thickness in order to withstand wave loads etc. Optimization-wise, it actually causes problems with the decreasing thickness because the optimized structures only need very low density material (approaching zero density) at the top. Impos- 
ing an extra load case mimicking wave loads at the top of the dam would make the problem much easier to solve, however, here we have avoided such a formulation in order to show the capability of the proposed method to solve problems where the fluid/void regions only are divided by a very weak structural region. The phase field approach [8] and the "filling-detection" scheme [6] have trouble solving the latter kind of problems.

\subsection{Three dimensions}

The proposed method can without further complications (except for the increased CPU-time) be extended to three dimensions. To demonstrate this, we show two examples: the internally pressurized lid and a dam design problem.

\subsubsection{Internally pressurized lid in 3D}

The design domain for the internally pressurized lid example is seen in Fig. 11a. The box drawn with solid lines indicates the design domain which has dimensions 2 by 2 by 1 and the box drawn with dashed lines constitutes the fixed pressure (non-design) domain. The design domain is simply supported along the thick solid line and the pressure domain is supported on the external faces. Due to symmetry we model only one fourth of the design domain with the appropriate boundary conditions for the symmetry faces.

The structure is discretized by cubic C- $8 / 1 / 8$ elements, thus the element density distribution is tri-linear with 6859 nodal design variables. The material Young's modulus is 1, Poisson's ratio is 0.3 and the fluid bulk modulus is $K_{\text {fluid }}=10$. The filter size was selected as 1.1 times the element size.

Different views of the optimized structure is seen in Figs. 11b, c and d. The initial compliance is $W_{\text {init }}=24.58$ and the final compliance is $W_{\text {final }}=0.734$. The dome shaped optimized topology is seen to be very similar to the topology obtained for the 2D case (Fig. 5c). By visual comparison it also seems to be very similar to the result obtained by the Aalborg group [5].

\subsection{D Dam}

In the final example, we design a 3-dimensional dam. The design domain shown in Fig. 12a has the dimensions 0.5 by 1 by 0.5 (indicated by solid lines) and there is a fixed fluid domain with the size 0.1 by 1 by 0.5 (indicated by dashed lines). The box is fully supported on the sides and on the bottom (indicated with thicker lines). Thus the design problem corresponds to finding the optimal dam in a rectangular shaped gorge. Due to symmetry the optimization is only performed on the half domain. Fig. 12b-e shows the optimized topology as voxel plots (all voxels with $\mu_{1}\left(1-\mu_{2}\right)>0.5$ are drawn) and as level surface 


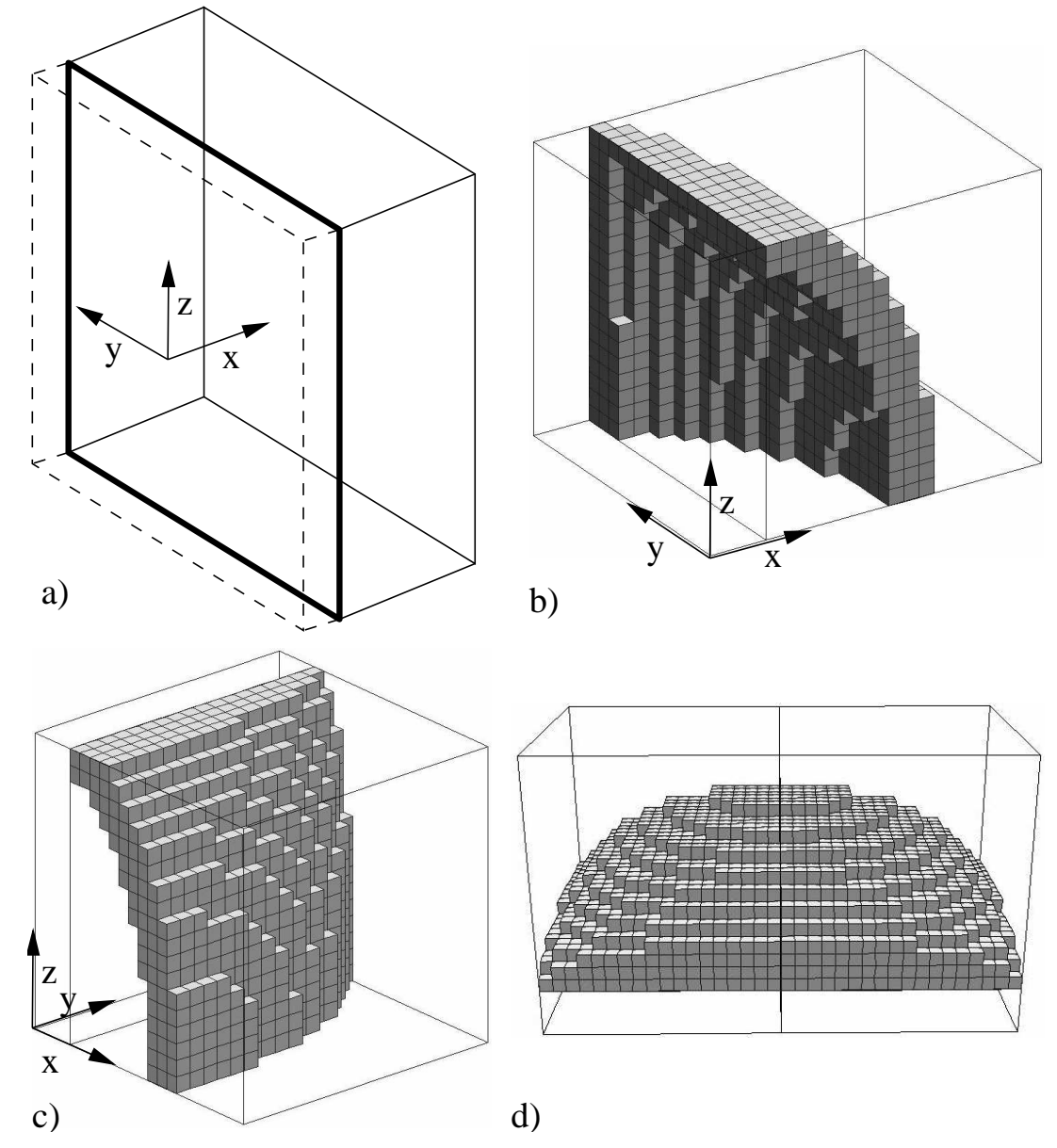

Fig. 11. a) Design domain and boundary conditions for internally loaded lid. b, c and d) different views of the optimized topology.

plots for $\mu_{1}\left(1-\mu_{2}\right)=0.5$ with supports indicated by black regions. The optimized dam structure is at the upper edge resembling a moon shape (as was expected). At the bottom the cross-section is cone shaped, indicating that also bending moments play a role in the optimized topology. It must be emphasized that this example only is intended for illustrative purposes. Real dam design require consideration of much more complicated loading and physics situations.

\section{Conclusions}

In this paper we have proposed a new method for handling pressure load problems in the density approach to topology optimization. The basic idea of the method is to define the void phase to be incompressible, i.e. as a hydrostatic fluid, and thereby we can transfer the pressure load through the fluid regions. In order to solve problems involving incompressibility we have to define the finite element problem in a mixed displacement/pressure form. This mixed 

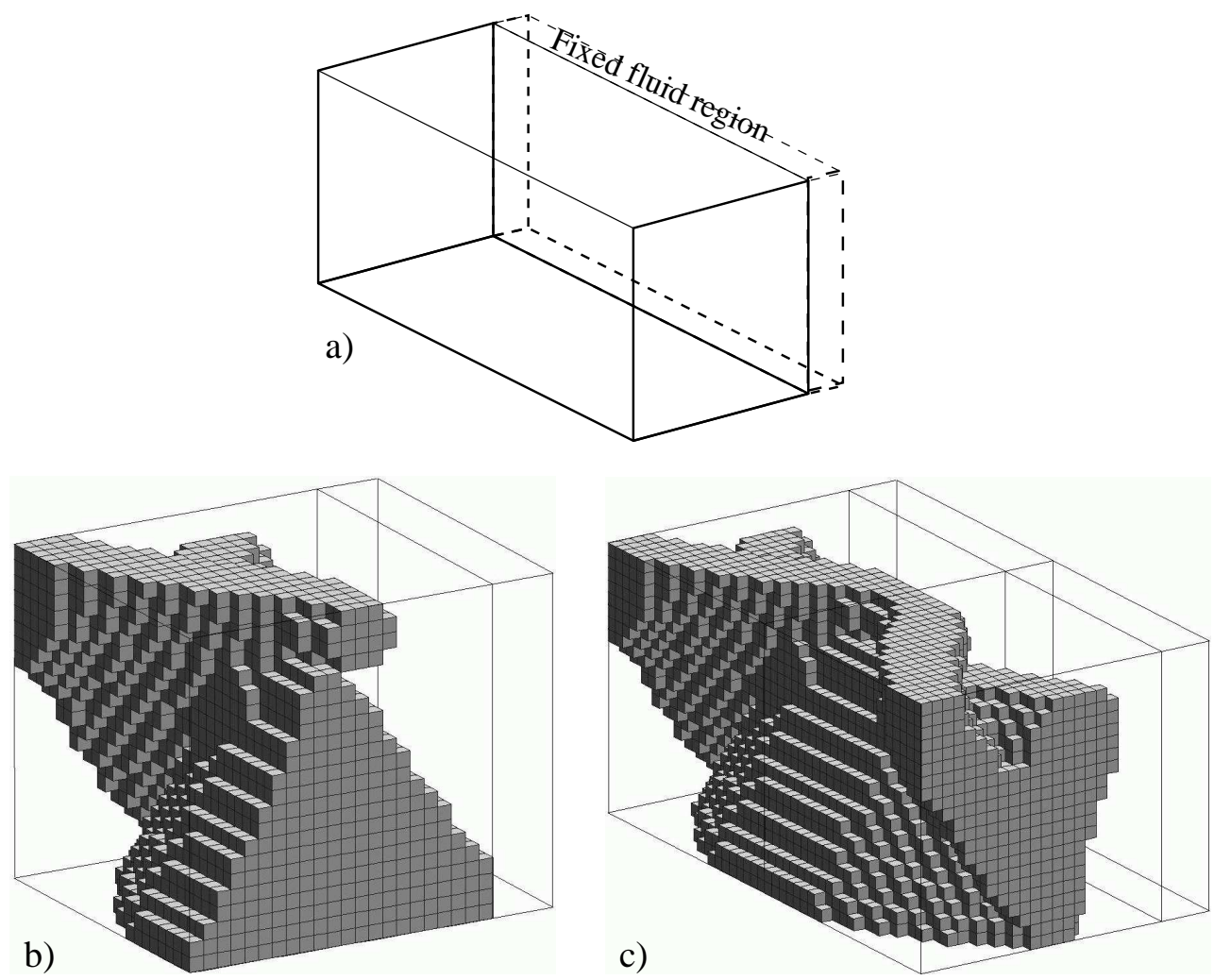

c)
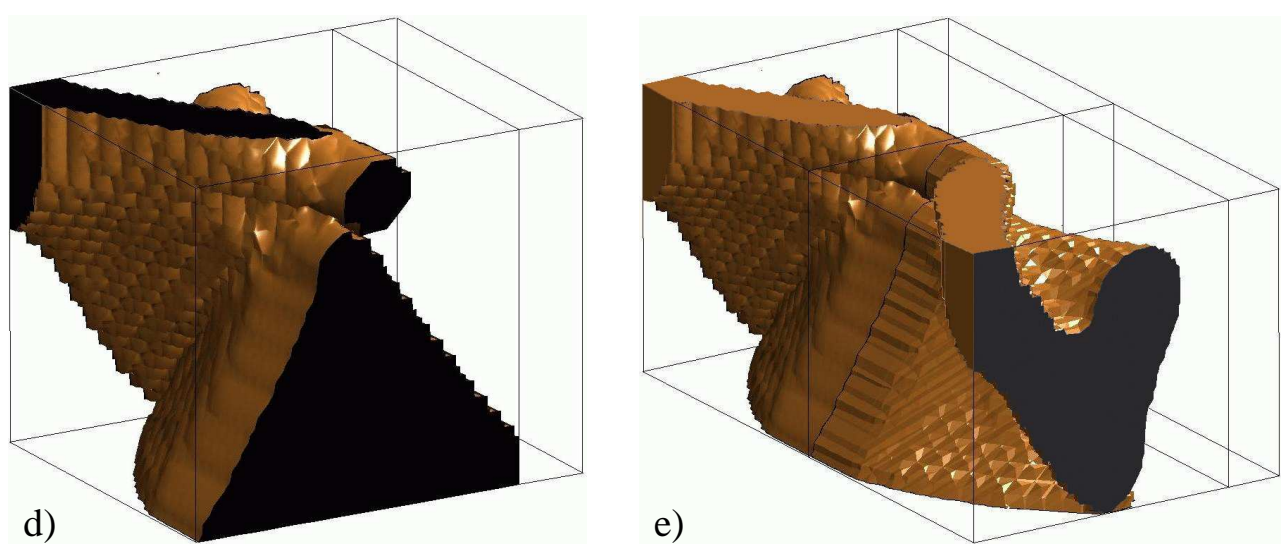

Fig. 12. a) Design domain and boundary conditions for dam. b-e) Different illustrations of the optimized topology. b) and c) show voxel representations and d) and e) show contour surfaces. b) and d) show half the structure (equal to the analysis domain) and c) and e) show the full domain.

form is a standard method in many FE-codes and should thus not complicate the implementation of the method. Otherwise, our method works exactly as the usual density based approaches - one only has to add a fixed fluid region to the design domain in order to impose the pressure loading through the fluid region. Introducing a second design variable into the formulation we can solve three phase (solid/fluid/void) problems as encountered in the design of dams and we can prevent internal cavities in becoming fluid filled by constraining 
the amount of fluid.

For the case of pure pressure load problems the convergence is very stable as long as we consider distribution of an incompressible elastic material. Here we typically obtain convergence (maximum change of design variables between two iterations less than 0.001) to nice black and white solutions in less than 100 iterations. For the case of distribution of compressible elastic material, the convergence is best if the fluid bulk modulus is at most 10-20 times larger than the bulk modulus of the elastic material. As demonstrated by several examples, however, the optimized topologies do not seem to change when the fluid bulk modulus is increased towards infinity thus the above limitation on the fluid bulk modulus does not constitute a problem.

For the design of dams, good convergence was difficult to obtain. However, it is well-known that self-weight problems are difficult to make converge (c.f. the discussion in [22]) and we thus attribute the problems to the self-weight issue and not to the proposed interpolation scheme. In order to improve the convergence of these problems one may have to use improved MMA approximations or globally convergent methods [22].

As demonstrated by the pressurized lid and dam examples the proposed scheme immediately applies to 3D problems. Here we only considered academic problems but it will be interesting to test the scheme on real life problems such as pressure vessels, pumps or other pressure loaded structures.

Finally, we note that the proposed mixed formulation can be extended to dynamic (acoustic-structure interaction) problems as discussed in refs. [27,28].

\section{Acknowledgments}

The support from the Danish Technical Research Council through the grant "Designing bandgap materials and structures with optimized dynamic properties", from Eurohorcs/ESF European Young Investigator Award (EURYI) through the grant "Synthesis and topology optimization of optomechanical systems" and from the Danish Center for Scientific Computing (DCSC) is gratefully acknowledged. Also the authors wishes to thank professors Jakob Søndergaard Jensen, Martin P. Bendsøe, Pauli Pedersen and other members from the TopOpt group (www.topopt.dtu.dk) for inspiring discussions on the presented work.

\section{References}

[1] M. P. Bendsøe, N. Kikuchi, Generating optimal topologies in structural design using a homogenization method, Computer Methods in Applied Mechanics and Engineering 71 (2) (1988) 197-224. 
[2] M. P. Bendsøe, O. Sigmund, Topology Optimization - Theory, Methods and Applications, Springer Verlag, Berlin Heidelberg, XIV+370 pp., 2003.

[3] V. B. Hammer, N. Olhoff, Topology optimization of continuum structures subjected to pressure loading, Structural and Multidisciplinary Optimization 19 (2000) 85-92.

[4] J. Du, N. Olhoff, Topological optimization of continuum structures with designdependent surface loading - Part I: New computational approach for 2D problems, Structural and Multidisciplinary Optimization 27 (2004) 151-165.

[5] J. Du, N. Olhoff, Topological optimization of continuum structures with designdependent surface loading - Part II: Algorithm and examples for 3D problems, Structural and Multidisciplinary Optimization 27 (2004) 166-177.

[6] B. Chen, N. Kikuchi, Topolgy optimization with design-dependent loads, Finite Element in Analysis and Design 37 (2001) 57-70.

[7] M. B. Fuchs, N. N. Y. Shemesh, Density-based topological design of structures subjected to water pressure using a parametric loading surface, Structural and Multidisciplinary Optimization 28 (1) (2004) 11-19.

[8] B. Bourdin, A. Chambolle, Design-dependent loads in topology optimization, ESAIM: Control, Optimisation and Calculus of Variations 9 (2003) 19-48.

[9] G. Allaire, F. Jouve, A.-M. Toader, Structural optimization using sensitivity analysis and a level-set method, Journal of Computational Physics 194 (1) (2004) 363-393.

[10] Z. Liu, J. G. Korvink, R. Huang, Structure topology optimization: fully coupled level set method via FEMLAB, Structural and Multidisciplinary Optimization 29 (6) (2005) 407-417.

[11] O. Sigmund, S. Torquato, Design of materials with extreme thermal expansion using a three-phase topology optimization method, Journal of the Mechanics and Physics of Solids 45 (6) (1997) 1037-1067.

[12] L. V. Gibiansky, O. Sigmund, Multiphase elastic composites with extremal bulk modulus, Journal of the Mechanics and Physics of Solids 48 (3) (2000) 461-498.

[13] O. Sigmund, Design of multiphysics actuators using topology optimization Part II: Two-material structures, Computer Methods in Applied Mechanics and Engineering 190 (49-50) (2001) 6605-6627.

[14] O. C. Zienkiewicz, R. L. Taylor, The Finite Element Method (Parts 1-3), Butterworth Heinemann, Oxford, 2000.

[15] C. S. Jog, R. B. Haber, Stability of finite element models for distributedparameter optimization and topology design, Computer Methods in Applied Mechanics and Engineering 130 (3-4) (1996) 203-226.

[16] O. Sigmund, On the design of compliant mechanisms using topology optimization, Mechanics of Structures and Machines 25 (4) (1997) 493-524. 
[17] M. P. Bendsøe, Optimal shape design as a material distribution problem, Structural Optimization 1 (1989) 193-202.

[18] M. Zhou, G. I. N. Rozvany, The COC algorithm, part II: Topological, geometry and generalized shape optimization, Computer Methods in Applied Mechanics and Engineering 89.

[19] H. P. Mlejnek, R. Schirrmacher, An engineering approach to optimal material distribution and shape finding, Computational Methods in Applied Mechanics and Engineering 106 (1993) 1-26.

[20] M. P. Bendsøe, O. Sigmund, Material interpolation schemes in topology optimization, Archives of Applied Mechanics 69 (9-10) (1999) 635-654.

[21] M. Stolpe, K. Svanberg, An alternative interpolation scheme for minimum compliance optimization, Structural and Multidisciplinary Optimization 22 (2) (2001) 116-124.

[22] M. Bruyneel, P. Duysinx, Note on topology optimization of continuum structures including self-weight, Structural and Multidisciplinary Optimization 29 (4) (2005) 245-256.

[23] N. L. Pedersen, Maximization of eigenvalues using topology optimization, Structural and Multidisciplinary Optimization 20 (1) (2000) 2-11.

[24] K. Svanberg, The Method of Moving Asymptotes - A new method for structural optimization, International Journal for Numerical Methods in Engineering 24 (1987) 359-373.

[25] L. H. Olesen, F. Okkels, H. Bruus, A high-level programming-language implementation of topology optimization applied to steady-state Navier-Stokes flow, International Journal for Numerical Methods in Engineering 65 (7) (2006) 975-1001.

URL $10.1002 / \mathrm{nme} .1468$

[26] O. Sigmund, J. Petersson, Numerical instabilities in topology optimization: A survey on procedures dealing with checkerboards, mesh-dependencies and local minima, Structural Optimization 16 (1) (1998) 68-75.

[27] G. H. Yoon, J. S. Jensen, O. Sigmund, Topology optimization for acoustic structure interaction problems, in: M. P. Bendsøe, N. Olhoff, O. Sigmund (Eds.), Topological design optimization of structures, machines and materials - status and perspectives, Solid Mechanics and its applications, Springer, 2005.

[28] G. H. Yoon, J. S. Jensen, O. Sigmund, Topology optimization for acoustic structure interaction problems, International Journal for Numerical Methods in EngineeringAccepted. 


\section{A Finite element implementation and sensitivity analysis}

Following $[14]^{5}$, the tensorial form of the equilibrium equations (7) and (8) can be written in matrix/vector form as

$$
\int_{\Omega} \delta \varepsilon^{T} \boldsymbol{D}_{d} \boldsymbol{\varepsilon} d \Omega-\int_{\Omega} \delta \boldsymbol{\varepsilon}^{T} \boldsymbol{m} p d \Omega-\int_{\Omega} \delta \boldsymbol{u}^{T} \boldsymbol{F} d \Omega-\int_{\Gamma_{T}} \delta \boldsymbol{u}^{T} \boldsymbol{T} d \Gamma=0
$$

and

$$
\int_{\Omega} \delta p\left(p / K+\boldsymbol{m}^{T} \boldsymbol{\varepsilon}\right) d \Omega=0
$$

respectively. Here, $\boldsymbol{m}=\{1,1,1,0,0,0\}^{T}$ in $3 \mathrm{D}, \boldsymbol{m}=\{1,1,0\}^{T}$ in $2 \mathrm{D}$, $\boldsymbol{D}_{d}=2 G\left(\boldsymbol{I}_{0}-\frac{1}{3} \boldsymbol{m} \boldsymbol{m}^{T}\right)$ in $3 \mathrm{D}, \boldsymbol{D}_{d}=2 G\left(\boldsymbol{I}_{0}-\frac{1}{2} \boldsymbol{m} \boldsymbol{m}^{T}\right)$ in $2 \mathrm{D}$, and $\boldsymbol{I}_{0}$ is a diagonal matrix with the entries $\frac{1}{2}\lceil 2,2,2,1,1,1\rfloor$ in $3 \mathrm{D}$ and $\frac{1}{2}\lceil 2,2,1\rfloor$ in $2 \mathrm{D}$.

Based on this notation, the linear finite element problem can be formulated as

$$
\left[\begin{array}{ll}
\mathbf{K} & \mathbf{C} \\
\mathbf{C}^{T} & \mathbf{D}
\end{array}\right]\left\{\begin{array}{l}
\mathbf{u} \\
\mathbf{p}
\end{array}\right\}=\left\{\begin{array}{l}
\mathbf{f} \\
\mathbf{0}
\end{array}\right\}
$$

where

$$
\left.\begin{array}{l}
\mathbf{K}=\sum_{e} \mathbf{K}_{e}=\sum_{e} \int_{\Omega_{e}} \mathbf{B}^{T} \boldsymbol{D}_{d} \mathbf{B} d \Omega \\
\mathbf{C}=\sum_{e} \mathbf{C}_{e}=\sum_{e} \int_{\Omega_{e}} \mathbf{B}^{T} \boldsymbol{m} \mathbf{N}_{p} d \Omega \\
\mathbf{D}=\sum_{e} \mathbf{D}_{e}=\sum_{e} \int_{\Omega_{e}} \mathbf{N}_{p}^{T} \frac{1}{K} \mathbf{N}_{p} d \Omega \\
\mathbf{f}=\sum_{e} \mathbf{f}_{e}=\sum_{e}\left(\int_{\Omega_{e}} \mathbf{N}_{u}^{T} \mathbf{F} d \Omega+\int_{\Gamma_{T, e}} \mathbf{N}_{u}^{T} \mathbf{T} d \Omega\right)
\end{array}\right\}
$$

Here, $\sum_{e}$ means summation over elements, $\mathbf{B}$ is the strain displacement matrix, $\mathbf{N}_{\mathbf{u}}$ is the displacement shape function matrix and $\mathbf{N}_{p}$ is the pressure shape function matrix.

As an example of how to perform the sensitivity analysis, we consider the case of piece-wise constant density distribution, no self weight and minimization

$\overline{5}$ except for the sign of the pressure 
of strain energy. The objective function (20) can in matrix vector form be written as

$$
W(\boldsymbol{\mu})=\frac{1}{2} \boldsymbol{u}^{T} \mathbf{K}(\boldsymbol{\mu}) \boldsymbol{u}=\sum_{e} \frac{1}{2} \boldsymbol{u}_{e}^{T} \mathbf{K}_{e}\left(\mu_{e}\right) \boldsymbol{u}_{e} .
$$

Taking the derivative with respect to a design variation we get

$$
\frac{\partial W}{\partial \mu_{e}}=\frac{1}{2} \boldsymbol{u}_{e}^{T} \frac{\partial \mathbf{K}_{e}}{\partial \mu_{e}} \boldsymbol{u}_{e}+\boldsymbol{u}_{e}^{T} \mathbf{K}_{e} \frac{\partial \mathbf{u}_{e}}{\partial \mu_{e}}
$$

where it was used that the stiffness matrix is symmetric.

Following the idea of adjoint sensitivity analysis, we add a zero term corresponding to a free Lagrangian multiplier vector times the derivative of the first row of the finite element equation (A.3) and reorder the terms, i.e.

$$
\begin{aligned}
\frac{\partial W}{\partial \mu_{e}} & =\frac{1}{2} \boldsymbol{u}_{e}^{T} \frac{\partial \mathbf{K}_{e}}{\partial \mu_{e}} \boldsymbol{u}_{e}+\boldsymbol{u}_{e}^{T} \mathbf{K}_{e} \frac{\partial \mathbf{u}_{e}}{\partial \mu_{e}}+\boldsymbol{\lambda}^{T}\left(\frac{\partial \mathbf{K}_{e}}{\partial \mu_{e}} \boldsymbol{u}_{e}+\mathbf{K}_{e} \frac{\partial \mathbf{u}_{e}}{\partial \mu_{e}}\right) \\
& =\left(\frac{1}{2} \boldsymbol{u}_{e}^{T}+\boldsymbol{\lambda}^{T}\right) \frac{\partial \mathbf{K}_{e}}{\partial \mu_{e}} \boldsymbol{u}_{e}+\left(\boldsymbol{u}_{e}^{T}+\boldsymbol{\lambda}^{T}\right) \mathbf{K}_{e} \frac{\partial \mathbf{u}_{e}}{\partial \mu_{e}}
\end{aligned}
$$

where it was used that the coupling matrix $\boldsymbol{C}$ is independent on the design. Since the Lagrangian multiplier vector can be chosen freely, we can eliminate the last term of (A.7) by choosing $\boldsymbol{\lambda}=-\boldsymbol{u}$ and the sensitivity expression thus simplifies to the well-known

$$
\frac{\partial W}{\partial \mu_{e}}=-\frac{1}{2} \boldsymbol{u}_{e}^{T} \frac{\partial \mathbf{K}_{e}}{\partial \mu_{e}} \boldsymbol{u}_{e}
$$

